\title{
Diffusion coefficients in a lamellar lyotropic phase: evidence for defects connecting the surfactant structure
}

\author{
Doru Constantin* and Patrick Oswald \\ École Normale Supérieure de Lyon, Laboratoire de Physique , 46 Allée d'Italie, 69364 Lyon Cedex 07, France
}

\begin{abstract}
We measure diffusion coefficients in the lamellar phase of the nonionic binary system $\mathrm{C}_{12} \mathrm{EO}_{6} / \mathrm{H}_{2} \mathrm{O}$ using fluorescence recovery after photobleaching (FRAP). The diffusion coefficient across the lamellae shows an abrupt increase upon approaching the lamellar-isotropic phase transition. We interpret this feature in terms of defects connecting the surfactant structure. An estimation of the defect density and of the variation in defect energy close to the transition is given in terms of a simple model.
\end{abstract}

PACS numbers: 61.30.Jf, 64.70.Md, 66.30.Jt

Topological defects are very important in condensed matter. In solids, most of the defects are out of equilibrium and form during growth or plastic deformation. In soft matter, like liquid crystals, defects sometimes nucleate spontaneously in great number. In this case, they often announce the transition towards a phase of higher symmetry.

Defective lamellar phases of lyotropic systems belong to this category. They contain structural defects that can be point-like or linear (dislocations). Unlike textural defects (e. $g$. focal conics), they are not visible in optical microscopy but they can be investigated using techniques such as FFEM (freeze-fracture electron microscopy) 1 [5],SANS 6], spin-labeling 7], birefringence measurements 2, 9], X-ray scattering [10, 11], NMR 12], etc. However, these methods do not give much information about the defect topology.

For instance, in lamellar phases, three elementary point defects are possible: "pores", "necks", and "passages" 13]. Necks connect the non-polar medium (surfactant structure) (fig.1a), pores (fig.1b) connect the polar medium (water), whereas passages join both media (fig.1c). Screw dislocations are also frequent because their energy is low [14]. These defects also connect both media and their core may be filled with the polar or the non-polar medium.

In this Letter we present a method of determining the topology of defects by monitoring the variation of the diffusion coefficients parallel $D_{\|}$and perpendicularly $D_{\perp}$ to the director (normal to the layers) of fluorescent probes that are dissolved either in the polar medium or in the non-polar one.

The system chosen is the lamellar phase of the lyotropic mixture of the non ionic surfactant $\mathrm{C}_{12} \mathrm{EO}_{6}$ with water. Spin-labeling measurements [7] have shown the existence of highly curved defects, the density of which abruptly increases a few degrees before melting. Dislocation loops perpendicular to the layers have been observed in FFEM [1, 2], but they can only account for a small fraction of the total defect density. In the following we present experimental results showing the existence of

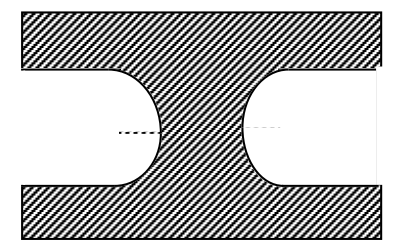

a)

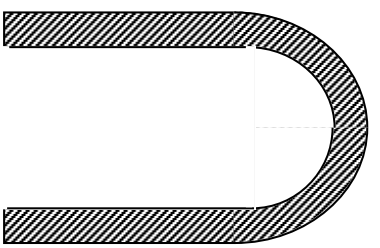

c)
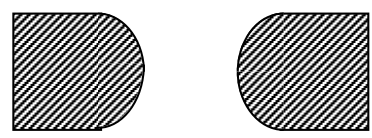

b)

FIG. 1: Elementary point defects in lamellar phases. a) "necks", b) - "pores", c) - "passages"

point defects. We confirm the existence of pores [7], and we also show that necks appear close to the lamellar isotropic transition.

We have first investigated the evolution of the diffusion coefficients for a fluorescent and hydrophobic dye. If $D_{\perp}$ (parallel to the layers) must not be significantly affected by the defects, $D_{\|}$(across the layers), which is very small for a perfect structure (the molecule has to cross a water barrier), should dramatically increase if the defects connect the surfactant structure. We performed these measures on planar domains (director parallel to the glass plates), in order to have access simultaneously to both $D_{\perp}$ and $D_{\|}$.

The surfactant was purchased from Nikko Ltd. and used without further purification. We use ultrapure water from Fluka. The surfactant concentration is $65 \%$ in weight. The dye (NBD-dioctylamin) is added in a concentration of about $0.1 \%$ and the mixture is then carefully homogenized. The samples are prepared between two parallel glass plates, with a spacing of $75 \mu \mathrm{m}$ and are 
sealed as described in detail elsewhere [9]. The lamellar phase is then oriented by use of the directional solidification technique [15]. We must emphasize that well oriented monodomains of millimetric size are needed for our measurements and, furthermore, we need planar anchoring on the plates. As a general rule, the lamellar phase prefers homeotropic anchoring (director orthogonal to the plates), but small and disorganized planar domains can be obtained by slowly cooling the isotropic phase of the mixture. We do that by moving the sample in the temperature gradient at about $30 \mu \mathrm{m} / \mathrm{s}$. The system is then allowed to reach equilibrium and we continue the process at $3 \mu \mathrm{m} / \mathrm{s}$. Oriented domains of the desired size are thus obtained. We found out that the planar anchoring is very much facilitated if the plates are ITO-covered.

Our measurement method for the diffusion constant is an adaptation of the technique known as fluorescence recovery after photobleaching (FRAP). The experimental setup was originally designed for the study of thin liquid films [16]. We focus the $\mathrm{TEM}_{00}$ mode of a multimode $\mathrm{Ar}^{+}$-ion laser (total power $70 \mathrm{~mW}$ ) on the sample, bleaching a spot about $40 \mu \mathrm{m}$ in diameter. The intensity profile of the beam is approximately Gaussian. Typical bleaching times are of the order of 5 seconds.

The evolution of the intensity profile (proportional to the concentration of non-bleached probe molecules $\left.c_{\mathrm{n}}(x, y, t)\right)$ is then monitored for one minute using a cooled CCD camera, capturing 30 images. During this time, the initial dark spot extends, due to the diffusion of bleached molecules. The concentration profile is elliptical because of the anisotropic diffusion. $D_{\perp}$ and $D_{\|}$are deduced from the images by fitting a Gaussian function. We will denote by $c(x, y, t)=c_{\text {tot }}-c_{\mathrm{n}}$ the concentration of bleached molecules. The bleaching is considered uniform across the sample, so the concentration obeys a twodimensional diffusion equation (in the plane of the sample); the $x$ axis is taken parallel to the director: $\mathbf{n} \| \mathbf{e}_{x}$. One has:

$$
c(x, y, t)=C \frac{\exp \left(-\frac{x^{2}}{a_{1}^{2}+4 D_{\|} t}-\frac{y^{2}}{a_{2}^{2}+4 D_{\perp} t}\right)}{\sqrt{\left(a_{1}^{2}+4 D_{\|} t\right)\left(a_{2}^{2}+4 D_{\perp} t\right)}}
$$

where $a_{1}$ and $a_{2}$ are the semi-axes of the initial bleaching spot. The quality of the fit is crucial, since the diffusion anisotropy is very high : $D_{\perp} \sim 100 D_{\|}$; reliable results can only be obtained with very well oriented planar domains : small departures from the elliptical shape (due to disorder) lead to relatively high variations in the determined value of $D_{\|}$. We must also be sure that the NBD molecules remain in the non-polar medium. This was shown by checking that, in the direct hexagonal phase of the same system, NBD molecules diffuse much faster along the cylinders than perpendicularly to them, whereas a hydrophilic dye diffuses isotropically (water forms a continuum among the cylinders).

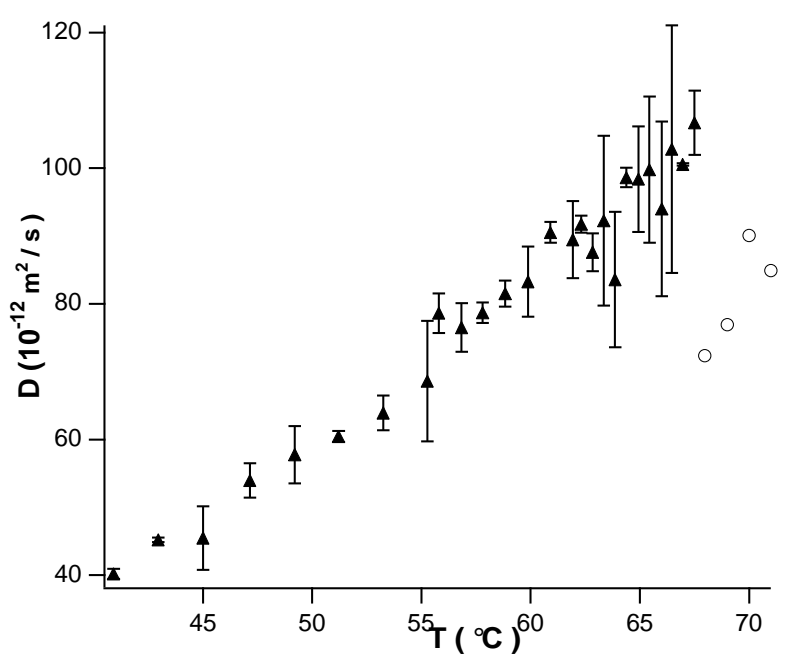

FIG. 2: NBD dioctylamin. $D_{\perp}$ in the lamellar phase (filled triangles) and $D$ in the isotropic phase (open circles). Where present, error bars are obtained by an average over three different measures

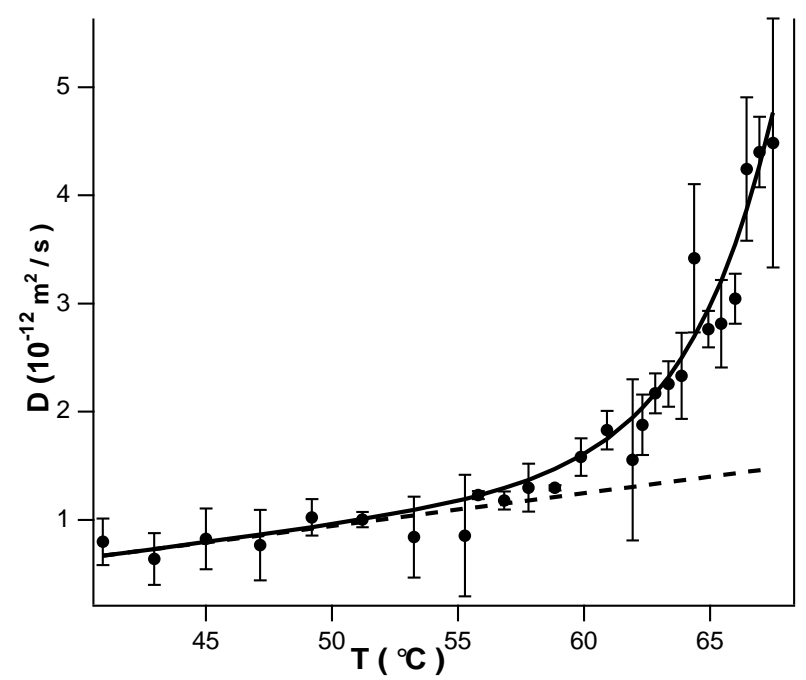

FIG. 3: $D_{\|}$of NBD dioctylamin. Axis on the right indicates the transition temperature. Error bars are obtained by an average over three different measures Dashed line represents linear extrapolation of low-temperature behaviour; solid line is exponential fit (see text).

Our data in the lamellar phase are displayed in Fig. 2 for $D_{\perp}$ and in Fig. 3 for $D_{\|} . D_{\perp}$ increases steadily with temperature from $4010^{-12} \mathrm{~m}^{2} / \mathrm{s}$ at $41^{\circ} \mathrm{C}$ to 107 $10^{-12} \mathrm{~m}^{2} / \mathrm{s}$ close to the transition temperature $\left(67.7^{\circ} \mathrm{C}\right)$. No pretransitional effect is observed.

The evolution of $D_{\|}$is completely different; it remains of the order of $10^{-12} \mathrm{~m}^{2} / \mathrm{s}$ until about $55^{\circ} \mathrm{C}$, when it starts to increase abruptly, reaching $510^{-12} \mathrm{~m}^{2} / \mathrm{s}$ at the 
transition. The low-temperature value could be due to probe molecules "jumping" the water barrier or to frozenin defects (the density of which does not depend on temperature), whereas the superimposed variation $\left(D_{\mathrm{def}}\right)$ clearly shows that bridges are formed between the surfactant layers, providing a passage for the molecules. We try now to characterize these passages as to their morphology and density.

One possibility would be that screw dislocations observed by FFEM provide passage for the molecules, but their density at the transition (about $100 \mu \mathrm{m}^{-2}$ ) [2], would only account for a small fraction of the measured $D_{\text {def }}$. Indeed, a simple model for the diffusion induced by the screw dislocations [17] gives :

$$
\frac{D_{\mathrm{def}}}{D_{\perp}} \sim\left(\frac{r_{c}}{\xi}\right)^{2}+6 \pi\left(\frac{b}{\xi}\right)^{2}\left[0.07+0.009 \ln \left(\xi / r_{c}\right)\right]
$$

where $b=50 \AA$ is the Burgers vector of the dislocations (equal to the lamellar periodicity $\ell$ ) [1], $r_{c}$ is the core radius (whose value is close to $b$ ) and $\xi=1500 \AA$ is the average distance between two dislocations of opposite sign. The first term on the r.h.s of eq.(2) corresponds to the "pipe" diffusion along the core of the dislocations which we assume to be filled with the nonpolar medium (water cores would not contribute to diffusion). The latter comes from the helical structure of the layers and from the fact that the molecules can pass from one lamella to another without jumping across the water barrier. This formula yields : $D_{\text {def }} / D_{\perp} \sim 310^{-3}$, about one order of magnitude less than the measured value for $D_{\text {def }} / D_{\perp} \sim 410^{-2}$. Hence, screw dislocations are certainly not the only type of defects present in the sample, conclusion that confirms EPR results. Consequently, we assume that the largest contribution to $D_{\text {def }}$ comes from point defects of type "neck" close to the transition.

We now estimate the number of defects needed in order to obtain the measured increase in $D_{\|}$. If the characteristic size of a defect is given (roughly) by the distance between layers $(\ell \sim 50 \AA)$, the induced diffusion coefficient $D_{\text {def }}$ along the director is then related to the defect density in the plane $n$ by :

$$
D_{\mathrm{def}} \simeq D_{\perp} n \ell^{2}
$$

within a numerical factor of order unity. Equation (3) simply states that, once a molecule has diffused to the space occupied by a defect, it crosses to the adjacent layer. Our data yield $n \simeq 0.09 \ell^{-2}=1200 \mu \mathrm{m}^{-2}$, signifying that about $10 \%$ of the molecules are in the defects just before the transition. This value is compatible with the increase of the molecule proportion in the defects found by EPR [7] within the five degrees preceding the transition and can also explain the birefringence drop observed in the same temperature range and previously interpreted in terms of disorientation of the lamellae induced by the proliferation of screw dislocation walls [2].

Since the defects are at thermal equilibrium, their number will be proportional to $\exp \left(-E_{\text {def }} / k_{B} T\right)$, where $E_{\text {def }}$ (the energy of the defect) decreases upon approaching the transition. The simplest assumption is that of linear behaviour :

$$
E_{\mathrm{def}}=\alpha\left(T_{0}-T\right)+E_{0}
$$

the fit is actually very good (figure 3) and gives a constant $\alpha \simeq 100 k_{B}$.

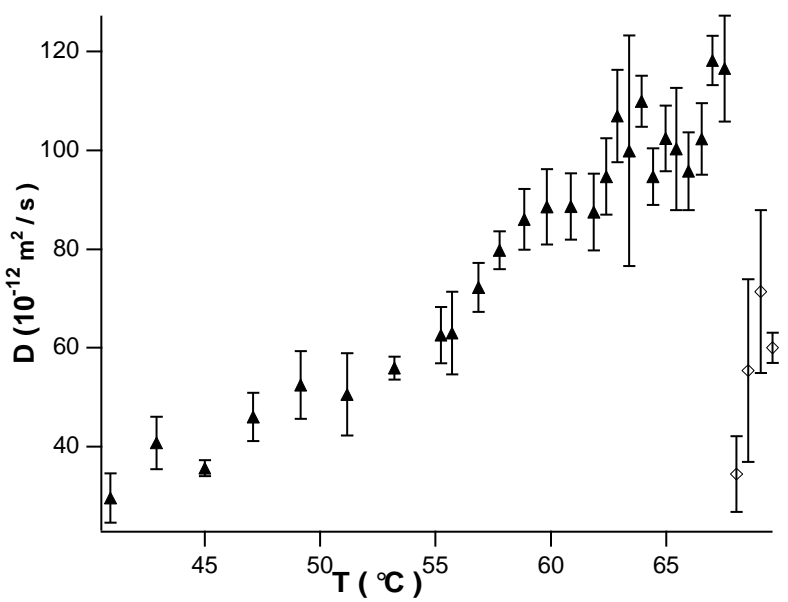

FIG. 4: Fluorescein. $D_{\perp}$ in the lamellar phase (filled triangles) and $D$ in the isotropic phase (open circles). Error bars are obtained by an average over three to five different measures

We have also performed a complementary measure, determining the diffusion coefficients of a hydrophilic dye (fluorescein) in the same temperature range. The fluorescein is less bleached by the laser beam, so the dark spot is much less contrasted than for the dioctylamin. A shorter measure time $(20 \mathrm{~s})$ has been used, and the data is more noisy; the general behaviour is, however, very clear : while $D_{\perp}$ has roughly the same value as that in the surfactant structure, $D_{\|}$is roughly constant when raising the temperature and shows no particular pre- transitional increase. This observation definitely rules out screw dislocations, as well as passages (fig. 1c), that connect both media (in which case we should observe the same type of pretransitionnal effect with both dyes).

However, the "necks" are not the only defects present in the sample. Indeed, fluorescein diffuses about 10 times faster than the dioctylamine across the structure, whereas they have practically the same $D_{\perp}$ at all temperatures. This suggests the existence of pores facilitating diffusion in the aqueous medium. Furthermore, the same argument that led to eq. (3) gives a pore density proportional to the ratio $D_{\|} / D_{\perp}$ for the hydrophilic dye, which 


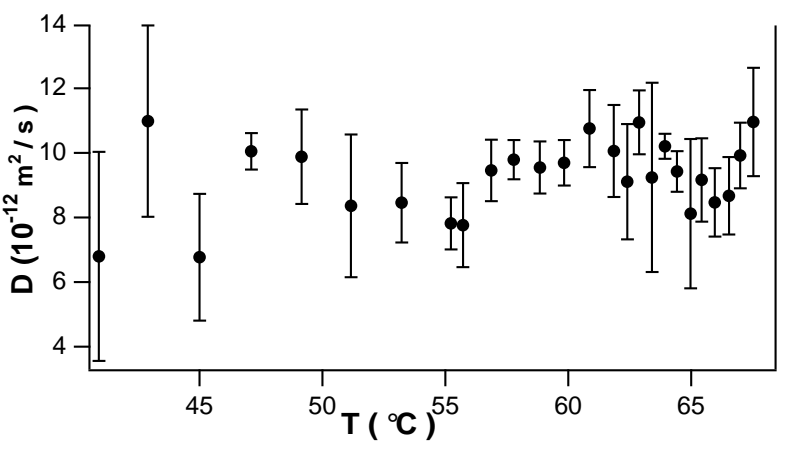

FIG. 5: $D_{\|}$of fluorescein. Axis on the right indicates the transition temperature. Error bars are obtained by an average over three to five different measures

would indicate that the number of pores decreases with temperature. This interpretation is coherent with EPR results 7] showing that the interfacial film suffers curvature inversion a few degrees before the transition, feature that can be interpreted as a sign of necks outnumbering pores.

One issue we have not tackled in this Letter and that will be the subject of future work is a microscopic model for the defect energy (eq. 41). Theoretical treatments 21, 22] have mainly focused upon dilute lamellar phases (while our system is, on the contrary, very concentrated) and upon a passage-like structure for the defect. While the passage is a defect of the bilayer, which has no spontaneous curvature, for a neck (or a pore), one has to consider the energy of the monolayer, the spontaneous curvature of which plays a very important role: positive and negative values lower the elastic energies of pores and necks, respectively [13]. Our results (necks appear at higher temperature than pores) are coherent with the general trend of non-ionic surfactants that the curvature decreases upon heating [23]. This effect has been recently measured for a variety of water/surfactant/alkane systems 24].

At this point we can also mention that necks or pores have also been encountered in numerical simulations of lamellar phases of ternary systems close to the transition to the microemulsion phase 25 27.

In conclusion, we have presented unambiguous experimental evidence that the non-polar medium in the lamellar phase of the lyotropic system $\mathrm{C}_{12} \mathrm{EO}_{6} / \mathrm{H}_{2} \mathrm{O}$ increases its connectivity when approaching the transition towards the isotropic phase, whereas the connectivity of the water (polar medium) decreases. These defects are certainly of the type "pores" at low temperature and of type "necks" at high temperature. These defects add to the screw dislocations already observed via FFEM, a conclusion that has already been drawn [2]. The new result is that our method allows us to distinguish between the different defect topologies, information which is difficult to obtain with other techniques.
Though the measures have been performed on one specific lyotropic mixture, we are confident that the FRAP measure of the diffusion coefficients can readily be applied to other similar systems to detect defects and their topological changes. We are currently using this method to characterize the defects appearing in the hexagonal phase of the same system close to the transition to the isotropic phase.

We acknowledge fruitful discussions with Thierry Biben.

* Electronic address: dcconsta@ens-lyon.fr

[1] M. Allain, Europhys. Lett. 2, 597 (1986).

[2] M. Allain, J. M. Di Meglio, Mol. Cryst. Liq. Cryst 124, 115 (1985).

[3] R. Strey et al., Langmuir 6, 1635 (1990).

[4] R. Strey et al., in Structure and Dynamics of Strongly Interacting Colloids and Supramolecular Aggregates in Solution, S.-H. Chen, J. S. Huang and P. Tartaglia (eds.) Kluwer, Dordrecht (1994).

[5] R. Strey et al., Ber. Bunssenges. Phys. Chem., 100, 182 (1996).

[6] M. C. Holmes, A. M. Smith and M. S. Leaver, J. Phys. II (France) 3, 1357 (1993).

[7] L. Paz et al., J. Phys. Chem. 88, 3415 (1984).

[8] M. Allain, P. Oswald, J. M. Di Meglio, Mol. Cryst. Liq. Cryst. 162B, 161 (1988).

[9] L. Sallen, P. Sotta and P. Oswald, J. Phys. Chem. B 101, 4875 (1997).

[10] Y. Rançon and J. Charvolin, J. Phys. Chem. 92, 6339 (1988).

[11] M. Clerc, A. M. Levelut and J. F. Sadoc, J. Phys. II (France) 1, 1263 (1991).

[12] W. Schnepp, S. Disch and C. Schmidt, Liq. Cryst. 14, 843 (1993).

[13] W. Helfrich, "Amphiphilic mesophases made of defects", in Physics of defects, eds. R. Balian, M. Kléman, J.-P. Poirier, North Holland (1980)

[14] M. Kléman, "Points, lines and Walls",J. Wiley, (1983).

[15] P. Oswald et al., J. Phys III 3, 1891 (1993).

[16] J.Bechhoefer et al., Phys. Rev. Lett. 79, 4922 (1997).

[17] P. Oswald, C. R. Acad. Sc. Paris 304, Série II, 1043 (1987)

[18] P. Oswald, M. Allain, J. Physique 46, 831 (1985).

[19] P. Oswald, M. Allain, J. Coll. Int. Sci., 126, 45 (1988).

[20] M. Allain, J. Phys. (France) 46, 225 (1985).

[21] L. Golubovic Phys. Rev. E 50, R2419 (1994).

[22] G. Gompper and J. Goos, J. Phys. II (France) 5, 621 (1995).

[23] J. N. Israelachvili, Intermolecular and Surface Forces, 2nd ed.; Academic Press, London (1983).

[24] T. Sottmann and R. Strey, J. Chem. Phys. 106, 8606 (1997).

[25] G. Gompper and D. M. Kroll, Phys. Rev. Lett. 81, 2284 (1998).

[26] R. Hołyst and W. T. Góźdź, J. Chem. Phys. 106, 4773 (1997).

[27] T. Biben and K. Helal, in preparation. 